\title{
TBOPP enhances the anticancer effect of cisplatin by inhibiting DOCK1 in renal cell carcinoma
}

\author{
WEI ZHANG ${ }^{1}$, XIAOXIAO ZHENG ${ }^{2}$, SHANGZHI XIE ${ }^{2}$, SHUFEN ZHANG ${ }^{2}$, JIAYAN MAO ${ }^{2}$, \\ YING CAI $^{2}$, XUEMEI LU ${ }^{2}$, WEI $\mathrm{CHEN}^{2}, \mathrm{HAIBIN} \mathrm{NI}^{3}$ and LIPING XIE ${ }^{1}$ \\ ${ }^{1}$ Department of Urology, The First Affiliated Hospital, School of Medicine, \\ Zhejiang University, Hangzhou, Zhejiang 310009; Departments of ${ }^{2}$ Medical Oncology and \\ ${ }^{3}$ General Surgery, Tongde Hospital of Zhejiang Province, Hangzhou, Zhejiang 310012, P.R. China
}

Received September 5, 2019; Accepted April 15, 2020

DOI: $10.3892 / \mathrm{mmr} .2020 .11243$

\begin{abstract}
The treatment of renal cell carcinoma (RCC) with chemotherapy remains a challenge; therefore, improving the knowledge of the molecular mechanisms underlying RCC chemoresistance and developing novel therapeutic strategies is important. Dedicator of cytokinesis 1 (DOCK1), the first member of the DOCK family to be discovered, displays various roles during tumorigenesis; however, its role during RCC progression is not completely understood. Therefore, the present study aimed to clarify the function of DOCK1 and 1-[2-(3'-(trifluorom ethyl)-(1,1'-biphenyl)-4-yl)-2-oxoethyl]-5-pyrrolidinylsulfonyl-2 $(1 \mathrm{H})$-pyridone (TBOPP), a DOCK1-sensitive inhibitor, during RCC development and chemoresistance. The results of CCK-8 and EdU assay indicated that TBOPP decreased RCC cell viability and proliferation compared with the control group, and sensitized RCC cells to cisplatin. Moreover, RCC cells with high DOCK1 expression levels displayed increased resistance to cisplatin, whereas DOCK1 knockdown enhanced the lethal effects of cisplatin on RCC cells. Furthermore, the results determined by western blotting, CCK- 8 and cell apoptosis assay indicated that TBOPP effectively reduced DOCK1 expression levels compared with the control group, and the
\end{abstract}

Correspondence to: Dr Liping Xie, Department of Urology, The First Affiliated Hospital, School of Medicine, Zhejiang University, 79 Qingchun Road, Hangzhou, Zhejiang 310009, P.R. China E-mail: xielp@zju.edu.cn

Dr Haibin Ni, Department of General Surgery, Tongde Hospital of Zhejiang Province, 234 Gucui Road, Hangzhou, Zhejiang 310012, P.R. China

E-mail: nihaibinpeter@163.com

Abbreviations: RCC, renal cell carcinoma; DOCK1, dedicator of cytokinesis 1; TBOPP, 1-[2-(3'-(trifluoromethyl)(1,1'-biphenyl)-4-yl)-2-oxoethyl]-5-pyrrolidinylsulfonyl2(1H)-pyridone; GEF, guanine nucleotide exchange factor; DHR-1, DOCK homology region-1; RAC1, RAC family small GTPase 1

Key words: renal cell carcinoma, DOCK1, TBOPP, chemoresistance
TBOPP-mediated cisplatin sensitizing effect was mediated by DOCK1 inhibition. The present study suggests that DOCK1 plays a vital role in RCC cell chemoresistance to cisplatin; therefore, TBOPP may serve as a novel therapeutic agent for RCC chemoresistance.

\section{Introduction}

Renal cell carcinoma ( $\mathrm{RCC}$ ) is a common cancer that accounts for $3 \%$ of all diagnosed human cases of cancer $(1,2)$. Surgery is the primary treatment strategy for localized RCC, but a third of patients with localized RCC experience metastases after nephrectomy (3). Chemotherapy is often an ineffective treatment strategy for RCC $(4,5)$. Although cisplatin is an effective therapeutic agent in a number of different types of cancer, RCC displays resistance to cisplatin (6), with only 4-6\% of patients with RCC responding to chemotherapy alone (7). Therefore, improving the knowledge of the molecular mechanisms underlying RCC chemoresistance and identifying novel $\mathrm{RCC}$ therapeutic targets is required.

The dedicator of cytokinesis (DOCK) family is one of the two members of the RAC guanine nucleotide exchange factor (GEF) family, which contain the evolutionarily conserved lipid-binding DOCK homology region-1 (DHR-1) domain and the catalytic DHR-2 domain (8). As a RAC-specific GEF, DOCK1 regulates phagocytosis, myoblastic fusion, cell migration and circular dorsal fold formation $(9,10)$. Abnormal expression and activity of DOCK1 is associated with the malignant characteristics of multiple tumors (11-14). Increasing evidence has indicated that DOCK1 regulates invasion and metastasis by acting on downstream receptor tyrosine kinases (15-17). Moreover, previous studies have revealed that DOCK1 expression levels correlate with chemoresistance in several types of cancer, such as bladder cancer and non-small-cell lung carcinomas $(12,18)$. However, the function of DOCK1 during RCC is not completely understood.

Considering the important role of DOCK1 during tumor development (11), DOCK1-selective inhibitors have been investigated. TBOPP [1-[2-(3'-(trifluoromethyl)-(1,1'-biphenyl)-4-yl)2-oxoethyl]-5-pyrrolidinylsulfonyl-2(1H)-pyridone] is a cellular activity inhibitor that binds to the DHR-2 domain of DOCK1 with high affinity, blocking its interaction with RAC family 
small GTPase 1 (RAC1) to inhibit its GEF activity without influencing the GEF activity of the diffuse B-cell lymphoma family, including $\mathrm{T}$ cell lymphoma invasion and metastasis 1 and Trio Rho guanine nucleotide exchange factor (19). TBOPP also displays an anti-invasion effect (20). However, the role of TBOPP during RCC has not been previously reported; therefore, the present study aimed to investigate the role of DOCK1 and TBOPP during RCC cisplatin resistance.

\section{Materials and methods}

Cell culture. The 786-O, ACHN, and Caki-1 cell lines were purchased from the American Type Culture Collection. 786-O cells were maintained in RPMI-1640 medium (Gibco; Thermo Fisher Scientific, Inc.) containing $10 \%$ fetal bovine serum (FBS; Gibco; Thermo Fisher Scientific, Inc.). Caki and ACHN cells were maintained in DMEM/F12 (Gibco; Thermo Fisher Scientific, Inc.) containing $10 \%$ FBS and $1 \%$ penicillin-streptomycin solution (Gibco; Thermo Fisher Scientific, Inc.). Cells were cultured at $37^{\circ} \mathrm{C}$ with $5 \% \mathrm{CO}_{2}$. TBOPP was purchased from WuXi AppTec and dissolved in DMSO before use. Cisplatin was purchased from Sigma-Aldrich, Merck KGaA. For single agent treatment groups, renal carcinoma cells were treated with $0,0.039,0.078,0.156,0.312,0.625,1.25,2.5,5$, 10,20 or $40 \mu \mathrm{M}$ TBOPP for $48 \mathrm{~h}$ at $37^{\circ} \mathrm{C}$, or $0,0.625,1.25,2.5$, 5 or $10 \mu \mathrm{M}$ cisplatin for $48 \mathrm{~h}$ at $37^{\circ} \mathrm{C}$. For the combined treatment group, renal carcinoma cells were treated with $10 \mu \mathrm{M}$ TBOPP and $0,0.625,1.25,2.5,5$ or $10 \mu \mathrm{M}$ cisplatin for $48 \mathrm{~h}$ at $37^{\circ} \mathrm{C}$.

Small interfering (si)RNA transfection. The DOCK1-specific and negative control siRNAs were designed by Shanghai GenePharma Co., Ltd. The sequences of the DOCK1 siRNAs were as follows: sc-35207A sense, GAGACAGAUUGGCUU UGAATT and antisense, UUCAAAGCCAAUCUGUCUCTT; sc-35207B sense, GAGAGAACCAUAUAUACAATT and antisense, UUGUAUAUAUGGUUCUCUCTT; and sc-35207C sense, CAGCAAACAUCAAGAGAUATT and antisense, UAU CUCUUGAUGUUUGCUGTT. The sequences of the negative control siRNA were as follows: Sense, UUCUCCGAACGU GUCACGUTT and antisense, ACGUGACACGUUCGGAGA ATT. Cells were transfected with $8 \mu \mathrm{M}$ DOCK1 siRNAs (the mix of three sequences) or negative control siRNAs using Lipofectamine ${ }^{\circledR} 2000$ (Invitrogen; Thermo Fisher Scientific, Inc.), according to the manufacturer's protocol. After $24 \mathrm{~h}$ transfection, the cells were used for subsequent experimentation.

Western blotting. Western blotting was performed according to a previously described protocol (21). Following $24 \mathrm{~h}$ treated with TBOPP or transfected with siRNA, the cells were collected, and total protein was extracted using RIPA lysis buffer (Beyotime Institute of Biotechnology). Total protein was quantified using a bicinchoninic acid assay kit (Applygen Technologies, Inc.). Equal amounts of proteins ( $40 \mu \mathrm{g}$ per lane) were separated via $10 \%$ SDS-PAGE and transferred to a PVDF membrane. Subsequently, the membrane was blocked with 5\% non-fat milk in TBS and $0.1 \%$ Tween-20 buffer for $1 \mathrm{~h}$ at room temperature. The membrane was incubated overnight at $4^{\circ} \mathrm{C}$ with primary antibodies targeted against: DOCK1 (dilution 1:1,000; cat. no. ab97325; Abcam) and GAPDH (1:1,000; cat. no. ab9485; Abcam). Following primary incubation, the membrane was incubated with horseradish peroxidase-conjugated secondary antibodies (dilution 1:500; cat. no. 7074; Cell Signaling Technology) at room temperature for $2 \mathrm{~h}$. Protein bands were visualized using a Pierce ${ }^{\mathrm{TM}}$ ECL kit according to the manufacturer's protocol (Thermo Fisher Scientific, Inc.). Protein expression was semi-quantified using ImageJ software (version 1.8.0; National Institutes of Health). GAPDH was used as the loading control.

Cell Counting Kit-8 (CCK-8) assay. Cell viability was assessed using the CCK-8 assay (Dojindo Molecular Technologies, Inc.), according to the manufacturer's protocol. Briefly, RCC cells treated with TBOPP or DOCK siRNA for $24 \mathrm{~h}$ were seeded (3x10 4 cells/well) into 96-well plates. Following treatment with cisplatin, $10 \mu \mathrm{l}$ CCK-8 solution was added to each well and incubated for $2 \mathrm{~h}$ at room temperature. The absorbance value of each well at a wavelength of $450 \mathrm{~nm}$ was measured using an MRX II microplate reader (Dynex Technologies).

5-Ethynyl-2'-deoxyuridine (EdU) assay. The Click-iTEdU Imaging kit (Invitrogen; Thermo Fisher Scientific, Inc.) was used for the EdU assay according to the manufacturer's protocol. Briefly, cells were seeded $\left(1 \times 10^{4}\right.$ cells/well) into a 96-well plate. Following treatment, cells were fixed with $4 \%$ formaldehyde for $30 \mathrm{~min}$, permeated with $0.5 \%$ Triton-X-100 for $10 \mathrm{~min}$ at room temperature and washed with PBS. Subsequently, $100 \mu \mathrm{l}$ EdU reagent was added to each well for $30 \mathrm{~min}$ at room temperature and cells were incubated with $100 \mu \mathrm{l}$ Hoechest 33342 for $10 \mathrm{~min}$ to label the nuclei. Cells were observed using a fluorescence microscope at x200 magnification.

Cell apoptosis determination by flow cytometry. Early and late apoptosis was detected using a FITC-conjugated Annexin-V and propidium iodide (PI) kit (BD Biosciences), according to the manufacturer's protocol. Cells $\left(2-3 \times 10^{5}\right)$ were washed twice with pre-chilled PBS and resuspended in $100 \mu \mathrm{l}$ binding buffer with $5 \mu \mathrm{l}$ FITC-conjugated Annexin-V for $30 \mathrm{~min}$ at room temperature in the dark. Subsequently, cells were incubated with $100 \mu \mathrm{l}$ PI for $5 \mathrm{~min}$ at room temperature and then $400 \mu \mathrm{l}$ binding buffer was added. Apoptotic cells were analyzed using a BD Laser II flow cytometer (BD Biosciences) and FlowJo software 7.6 (FlowJo LLC).

Statistical analysis. Statistical analyses were performed using SPSS software (version 16.0; SPSS, Inc.) and GraphPad Prism (version 7; GraphPad Software, Inc.). Statistical differences between two groups were analyzed using a Student's t-test. Statistical differences among multiple groups were analyzed by one-way ANOVA followed by Tukey's post hoc test. Data are presented as the mean \pm standard deviation of three independent experiments. $\mathrm{P}<0.05$ was considered to indicate a statistically significant difference.

\section{Results}

TBOPP decreases $R C C$ cell proliferation and viability. To investigate the function of TBOPP, RCC cells were treated with different concentrations $(0-40 \mu \mathrm{M})$ of TBOPP and cell 
A

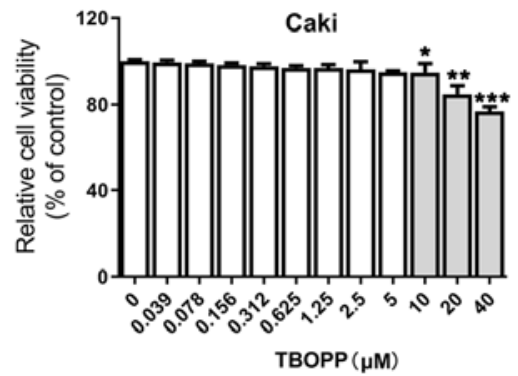

$\mathrm{B} \underline{\mathrm{Ca}}$

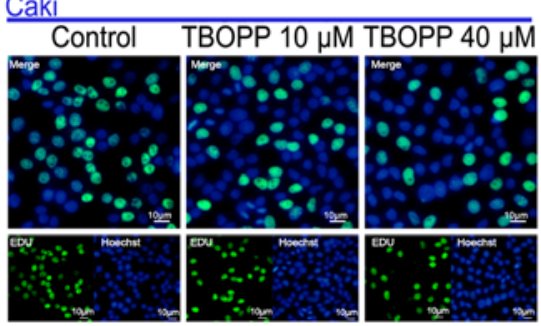

Caki

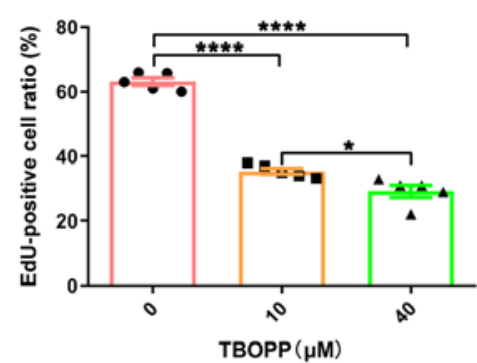

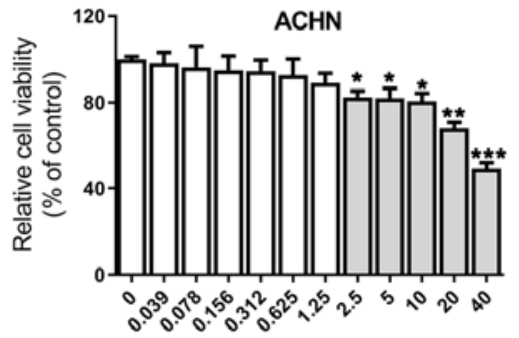

$\operatorname{TBOPP}(\mu \mathrm{M})$

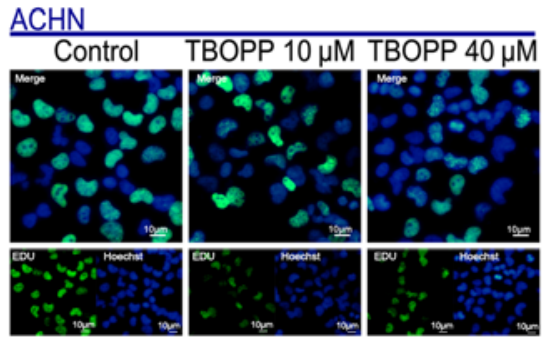

ACHN

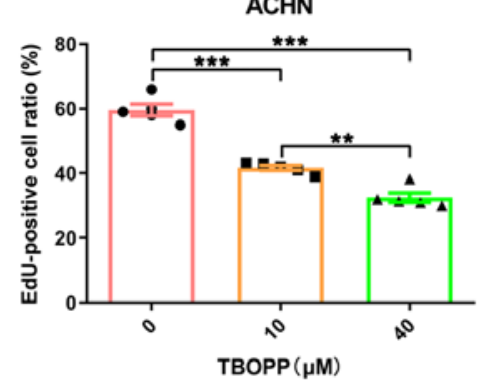

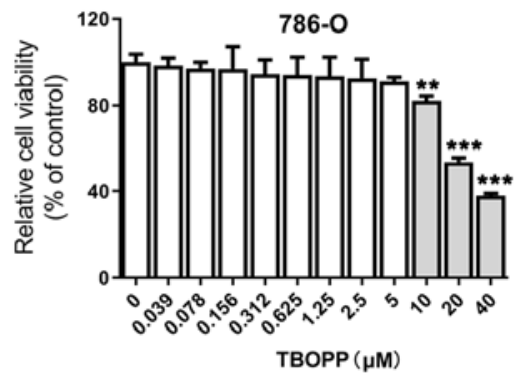

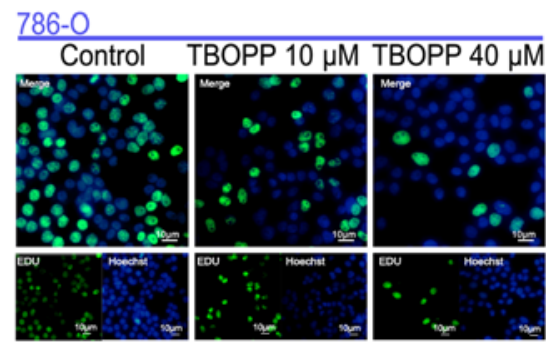

$786-0$

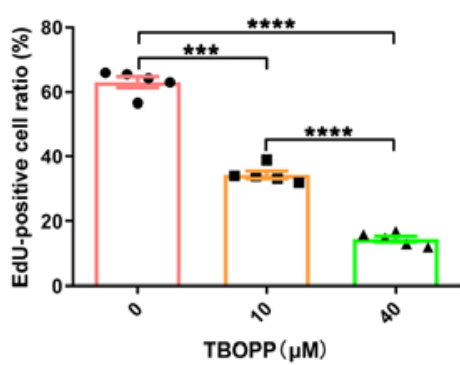

Figure 1. TBOPP reduces RCC cell viability and proliferation. (A) RCC cell viability was quantified using the Cell Counting Kit-8 assay. Grey columns indicate that there was a significant difference in cell viability compared with the control group. ${ }^{*} \mathrm{P}<0.05,{ }^{* * *} \mathrm{P}<0.001$ and ${ }^{* * * *} \mathrm{P}<0.001$ vs. the control group. (B) RCC cell proliferation was assessed using the EdU assay. Scale bar, $10 \mu \mathrm{m}$. The rate of EdU-positive cells in the $\mathrm{S}$ phase is presented. $\mathrm{P}<0.05$, ${ }^{* *} \mathrm{P}<0.01$, ${ }^{* * *} \mathrm{P}<0.001$ and ${ }^{* * * *} \mathrm{P}<0.0001$ as indicated. TBOPP, 1-[2-(3'-(trifluoromethyl)-(1,1'-biphenyl)-4-yl)-2-oxoethyl]-5-pyrrolidinylsulfonyl-2(1H)-pyridone; RCC, renal cell carcinoma; EdU, 5-Ethynyl-2'-deoxyuridine.

viability was assessed using a CCK- 8 assay. TBOPP decreased RCC cell viability from $10 \mu \mathrm{M}$ in a concentration-dependent manner (Fig. 1A). Subsequently, cells treated with two concentrations of TBOPP (10 and $40 \mu \mathrm{M})$ were assessed using the EdU assay. The results suggested that cell proliferation was also significantly decreased in the TBOPP treatment groups compared with the control group (Fig. 1B). The results demonstrated the anti-RCC function of TBOPP; $10 \mu \mathrm{M}$ TBOPP significantly reduced cell viability in all 3 cell lines and also significantly decreased cell proliferation in all 3 cell lines; thus, $10 \mu \mathrm{M}$ TBOPP was used for subsequent experiments.

TBOPP inhibits RCC cell cisplatin resistance. The effect of TBOPP on cisplatin resistance in RCC was investigated. TBOPP combined with cisplatin further reduced RCC cell viability compared with cisplatin alone (Fig. 2A). The EdU assay suggested that TBOPP significantly enhanced the antiproliferative activity of cisplatin in RCC cells (Fig. 2B). Moreover, TBOPP significantly increased cisplatin-induced RCC cell apoptosis (Fig. 2C). Collectively, the results demonstrated that TBOPP inhibited RCC cell cisplatin resistance.

DOCK1 knockdown sensitizes RCC cells to cisplatin. Subsequently, the role of DOCK1 during RCC was investigated. Firstly, the expression of DOCK1 in the three RCC cell lines was measured. DOCK1 expression levels were highest in the
Caki cell line, followed by the ACHN cell line and the 786-O cell line (Fig. 3A). The CCK-8 assay indicated that following treatment with cisplatin, the Caki cell line displayed the highest cell viability among the three $\mathrm{RCC}$ cell lines assessed $\left(\mathrm{IC}_{50}\right.$ values: Caki, $7.804 \mu \mathrm{M}$; ACHIN, 5.105 $\mu \mathrm{M}$; 786-O: $3.770 \mu \mathrm{M}$ ) (Fig. 3B). To further verify the role of DOCK1 during RCC, a DOCK1 siRNA was used to significantly knockdown DOCK1 expression, which was confirmed by western blotting (Fig. 3C). The CCK-8 assay suggested that DOCK1 knockdown significantly enhanced cisplatin-mediated reductions in cell viability (Fig. 3D). Additionally, flow cytometry analysis of apoptosis demonstrated that DOCK1 knockdown promoted cisplatin-induced apoptosis (Fig. 3E). Furthermore, the EdU assay indicated that following treatment with cisplatin, the proliferation of DOCK1 siRNA-treated cells was significantly lower compared with the control cells (Fig. 3F). The results suggest that DOCK1 promotes RCC cell cisplatin resistance.

TBOPP function during RCC is mediated by DOCK1. TBOPP is an inhibitor of DOCK1; therefore, to explore the relationship between TBOPP and DOCK1 during RCC, the inhibitory role of TBOPP on DOCK1 expression in RCC cells was assessed by western blotting. TBOPP significantly decreased DOCK1 expression levels compared with the control group (Fig. 4). Subsequently, the cell viability of cells co-treated with cisplatin and DOCK1 siRNA alone or DOCK1 siRNA 
A

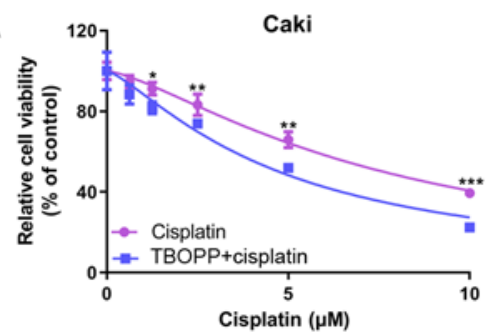

$\mathrm{B}$
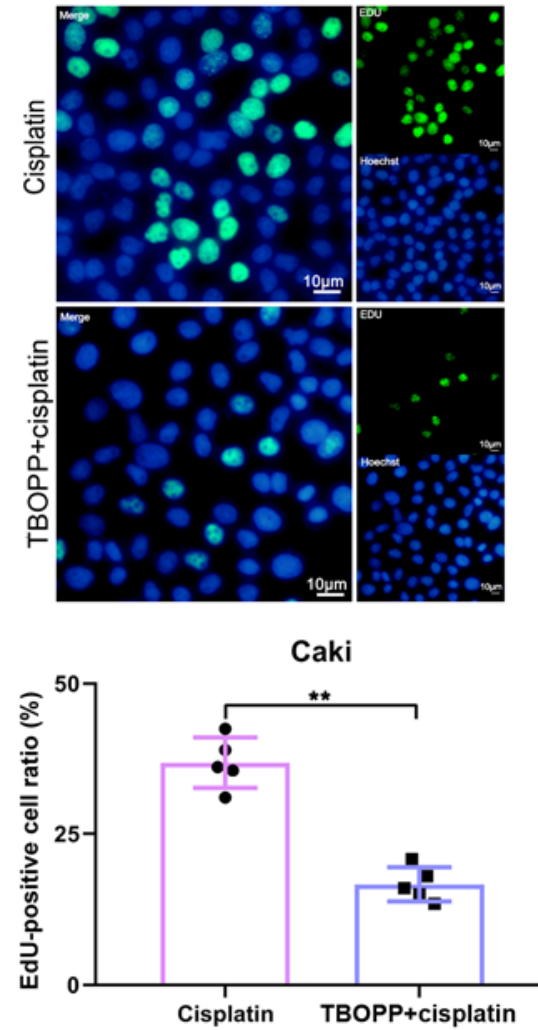

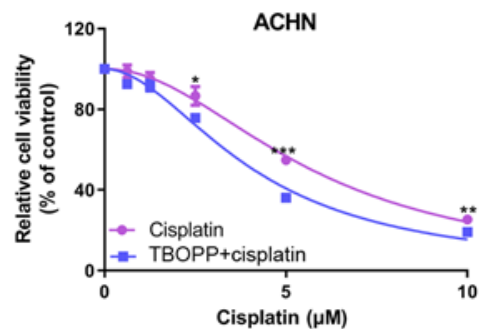

\section{ACHN}
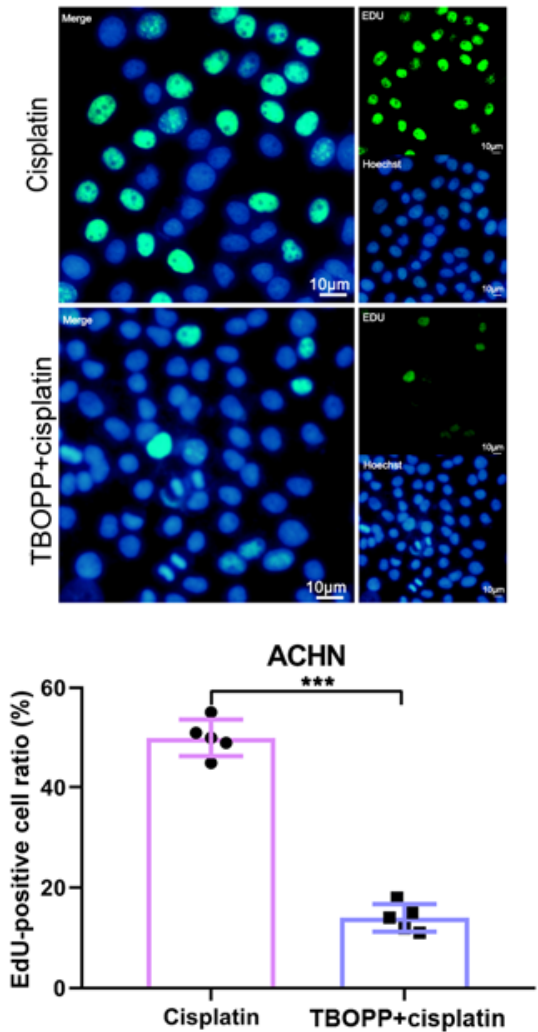

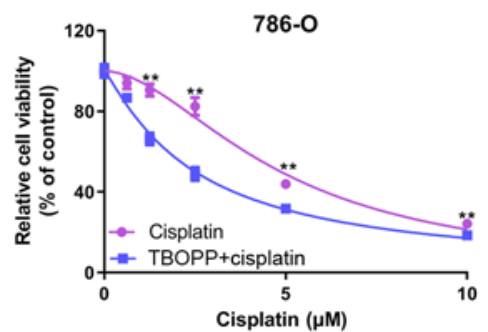

786-0
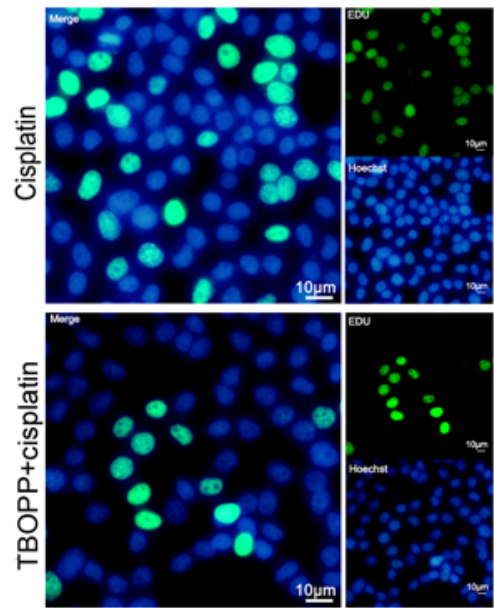

786-0

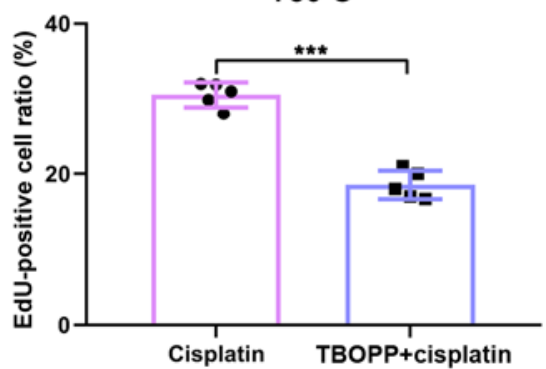

C
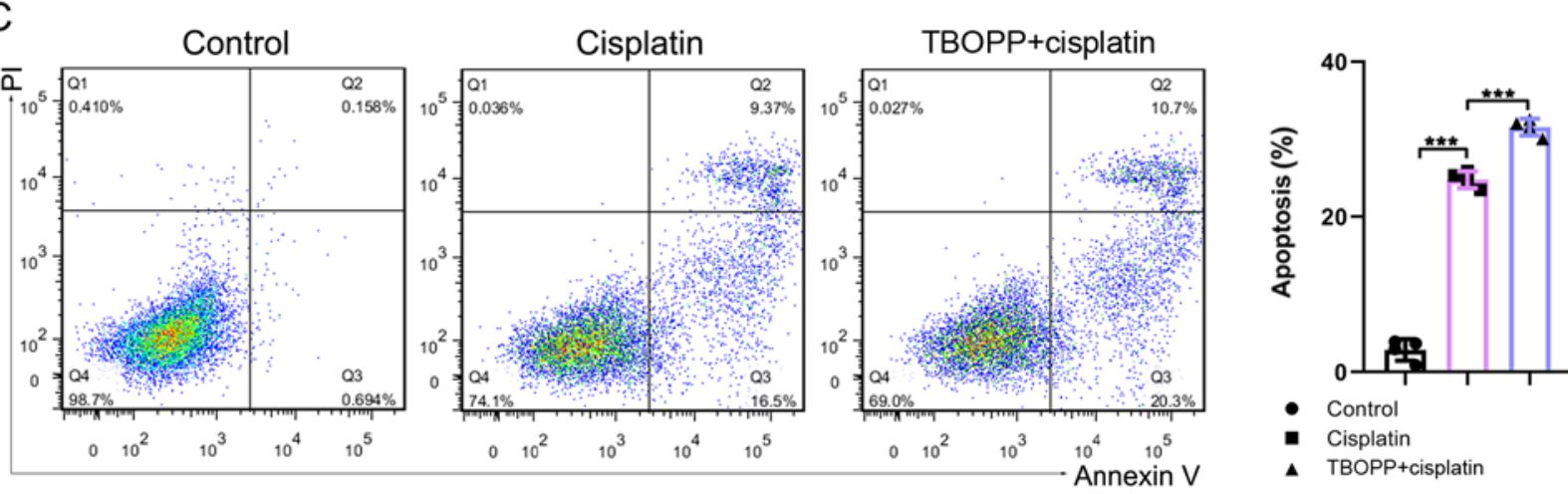

Figure 2. TBOPP sensitizes RCC cells to cisplatin. (A) RCC cell viability following treatment with cisplatin alone or cisplatin plus $10 \mu \mathrm{M}$ TBOPP, as determined by the Cell Counting Kit- 8 assay. ${ }^{*} \mathrm{P}<0.05,{ }^{* *} \mathrm{P}<0.01$ and ${ }^{* * *} \mathrm{P}<0.001$ vs. the TBOPP + cisplatin group. (B) RCC cell proliferation following treatment with cisplatin alone or cisplatin plus $10 \mu \mathrm{M}$ TBOPP, as determined by the EdU assay. Scale bar, $10 \mu \mathrm{m}$. The rate of EdU-positive cells in the S phase is presented. ${ }^{* *} \mathrm{P}<0.01$ and ${ }^{* * * *} \mathrm{P}<0.001$, as indicated. (C) Flow cytometric analysis of RCC cell apoptosis following treatment with cisplatin alone or cisplatin plus $10 \mu \mathrm{M}$ TBOPP. Cells in quadrants 2 and 3 were considered to be apoptotic. ${ }^{* * *} \mathrm{P}<0.001$, as indicated. TBOPP, 1-[2-(3'-(trifluoromethyl)-(1,1'-biphenyl)-4-yl)-2-oxoeth yl]-5-pyrrolidinylsulfonyl-2(1H)-pyridone; RCC, renal cell carcinoma; EdU, 5-Ethynyl-2'-deoxyuridine; PI, propidium iodide.

plus TBOPP was assessed by the CCK-8 assay. Cell viability in the DOCK1 siRNA group and the DOCK1 siRNA plus TBOPP group was not significantly different (Fig. 5A), which indicated that TBOPP-mediated cisplatin sensitivity did not occur in the absence of DOCK1 expression. The relationship between TBOPP and DOCK1 during RCC was further indicated by the results of the flow cytometry analysis of cell apoptosis (Fig. 5B). Transfection efficiency of DOCK1 siRNA 
A
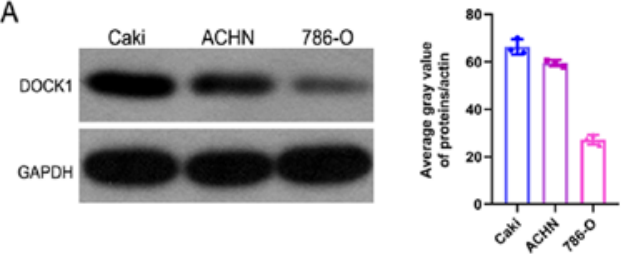

C

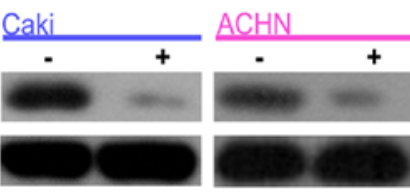

786-0
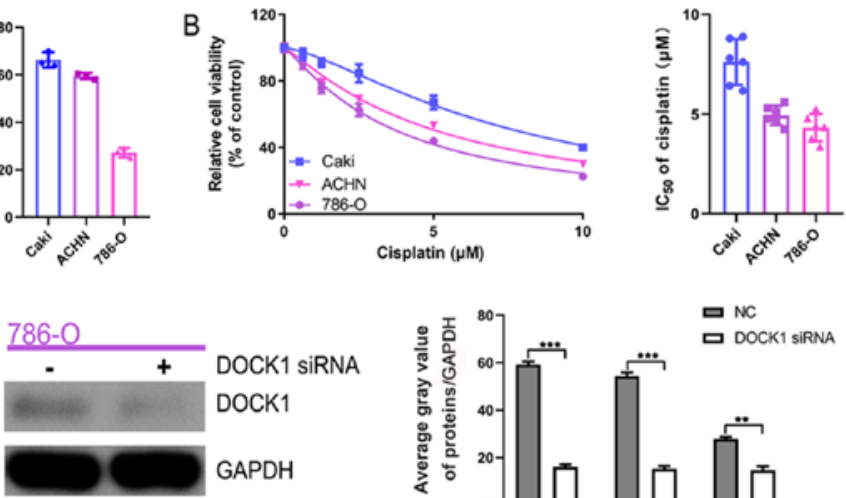

DOCK1 SiRNA DOCK1

GAPDH

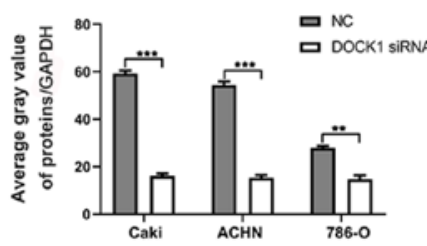

D
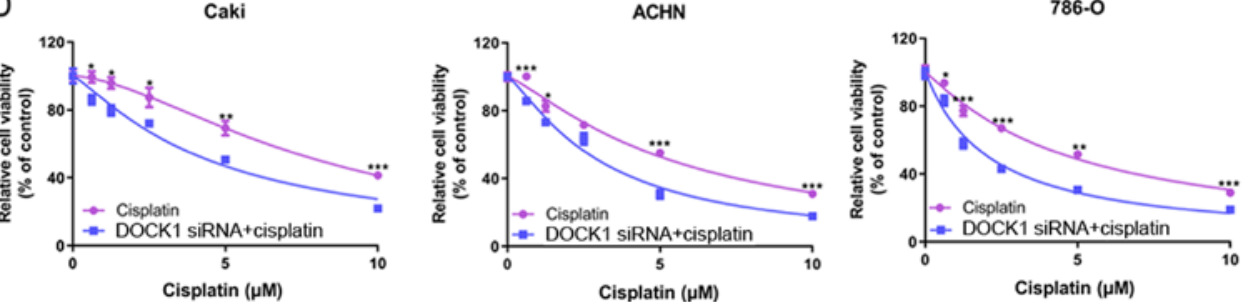

E
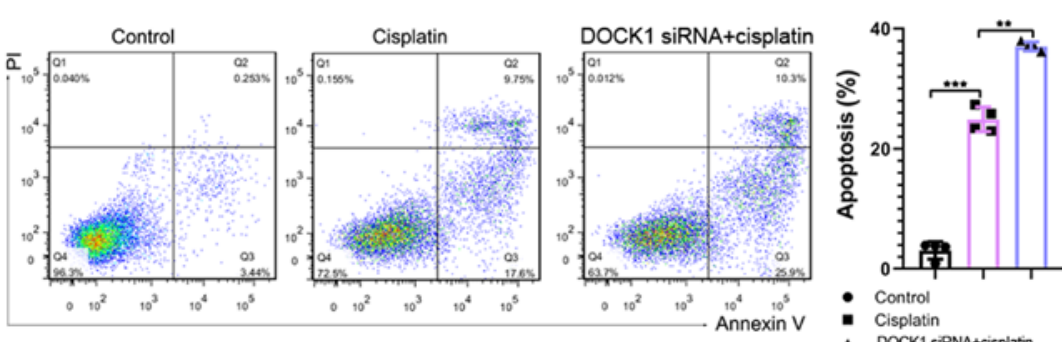

$\mathrm{F}$
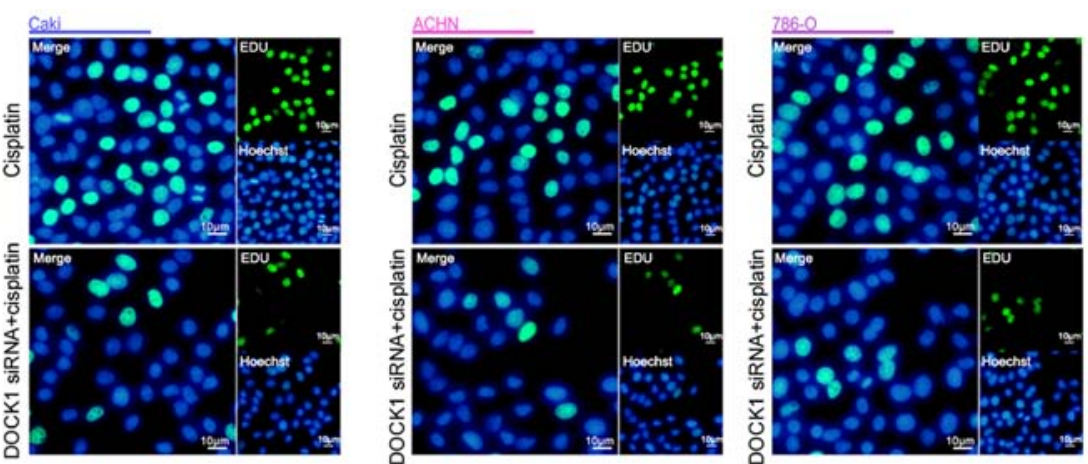

- Cisplatin

- DOCK1 siRNA+cisplatin
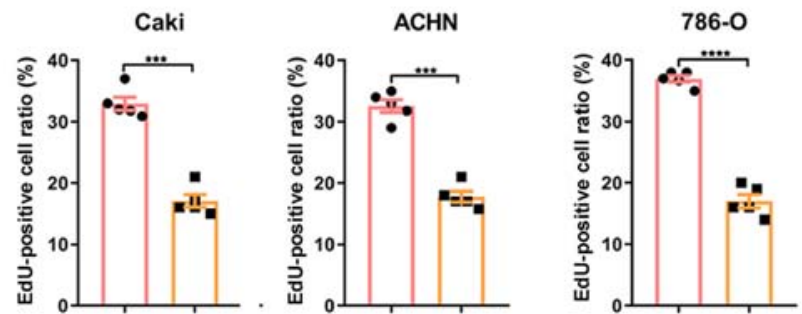

Figure 3. DOCK1 knockdown enhances cisplatin sensitivity during RCC. (A) DOCK1 expression levels in the three RCC cell lines were determined by western blotting. (B) The CCK-8 assay was performed to determine cell viability and the $\mathrm{IC}_{50}$ value of cisplatin in the three RCC cell lines. (C) The transfection efficiency of DOCK1 siRNA (the mix of three sequences) was assessed by western blotting. ${ }^{* *} \mathrm{P}<0.01$ and ${ }^{* * *} \mathrm{P}<0.001$, as indicated. (D) The CCK-8 assay was performed to determine cell viability in the three RCC cell lines following co-treatment with cisplatin and DOCK1 siRNA or negative control siRNA. " $\mathrm{P}<0.05$, ${ }^{* *} \mathrm{P}<0.01$ and ${ }^{* * * *} \mathrm{P}<0.001$ vs. the cisplatin group. (E) Flow cytometry analysis of RCC cell apoptosis following co-treatment with cisplatin and DOCK1 siRNA or negative control siRNA. ${ }^{* *} \mathrm{P}<0.01$ and ${ }^{* * *} \mathrm{P}<0.001$, as indicated. (F) RCC cell proliferation was assessed by the EdU assay following co-treatment with cisplatin and DOCK1 siRNA or negative control siRNA. Scale bar, $10 \mu \mathrm{m} .{ }^{* * * *} \mathrm{P}<0.001$ and ${ }^{* * * * *} \mathrm{P}<0.0001$, as indicated. DOCK1, dedicator of cytokinesis $1 ;$ RCC, renal cell carcinoma; CCK-8, Cell Counting Kit-8; siRNA, small interfering RNA; EdU, 5-Ethynyl-2'-deoxyuridine; NC, negative control; PI, propidium iodide. 

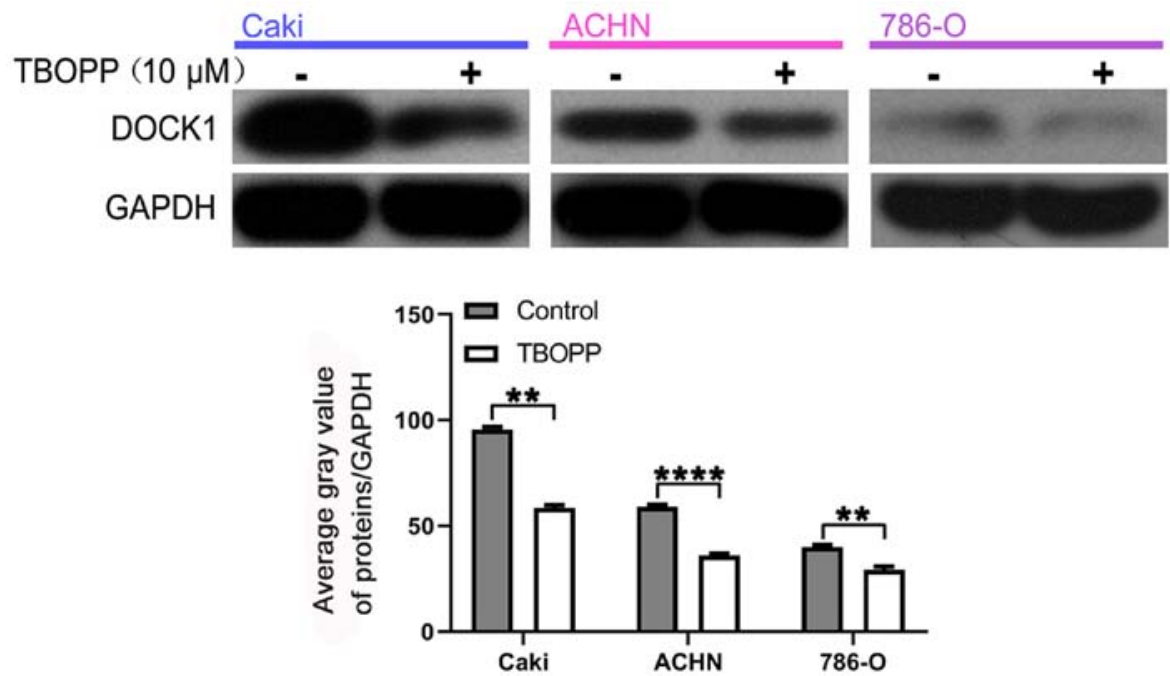

Figure 4. TBOPP inhibits DOCK1 expression. The inhibitory effect of TBOPP on DOCK1 expression levels was assessed by western blotting. ** $\mathrm{P}<0.01$ and ${ }^{* * * * *} \mathrm{P}<0.0001$, as indicated. TBOPP, 1-[2-(3'-(trifluoromethyl)-(1,1'-biphenyl)-4-yl)-2-oxoethyl]-5-pyrrolidinylsulfonyl-2(1H)-pyridone; DOCK1, dedicator of cytokinesis 1.
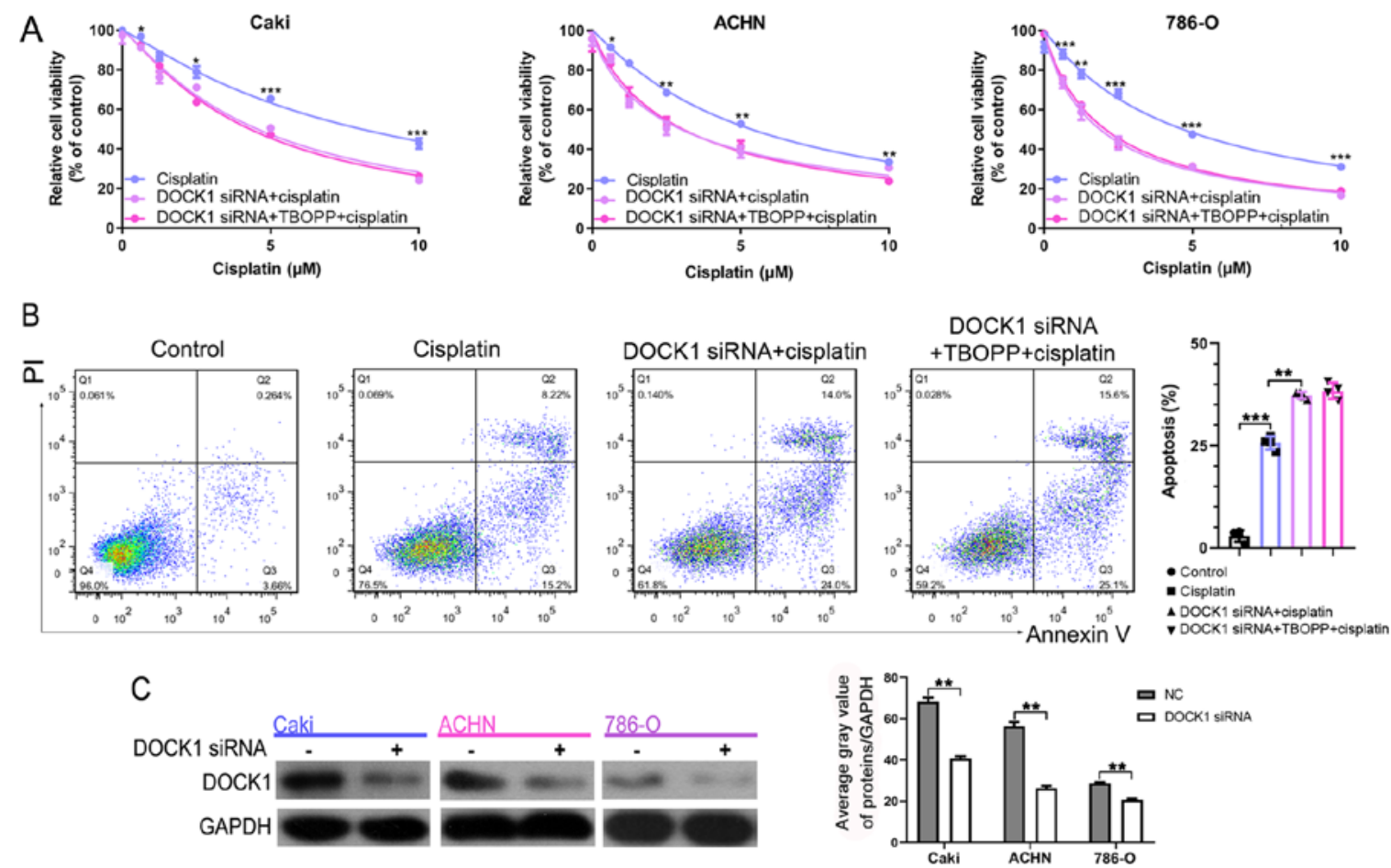

Figure 5. TBOPP enhances cisplatin sensitivity by inhibiting DOCK1. (A) RCC cell viability following co-treatment with cisplatin and DOCK1 siRNA or DOCK1 siRNA plus $10 \mu \mathrm{M}$ TBOPP. ${ }^{*} \mathrm{P}<0.05,{ }^{* *} \mathrm{P}<0.01$ and ${ }^{* * * *} \mathrm{P}<0.001$ vs. TBOPP + cisplatin. (B) Flow cytometry analysis of RCC cell apoptosis following co-treatment with cisplatin and DOCK1 siRNA or DOCK1 siRNA plus $10 \mu \mathrm{M}$ TBOPP. ${ }^{* *} \mathrm{P}<0.01$ and ${ }^{* * *} \mathrm{P}<0.001$, as indicated. (C) The transfection efficiency of DOCK1 siRNA (the mix of three sequences) was assessed by western blotting. ${ }^{* *} \mathrm{P}<0.01$, as indicated. TBOPP, 1-[2-(3'-(trifluoromethyl)-(1,1'-biphenyl)-4yl)-2-oxoethyl]-5-pyrrolidinylsulfonyl-2(1H)-pyridone; DOCK1, dedicator of cytokinesis 1; RCC, renal cell carcinoma; siRNA, small interfering RNA; NC, negative control; PI, propidium iodide.

was confirmed by western blotting (Fig. 5C). The results demonstrated that DOCK1 was involved in TBOPP-mediated cisplatin sensitivity.

\section{Discussion}

In patients with RCC, infrequent responses due to intrinsic and acquired chemoresistance significantly limit the clinical use of chemotherapeutic agents (22). Therefore, identifying the mechanisms underlying RCC chemoresistance and developing novel therapeutic strategies to resensitize RCC to anticancer drugs is important. The present study aimed to investigate the role of DOCK1 and its inhibitor TBOPP during RCC chemoresistance. The results suggested that TBOPP and DOCK1 may serve as therapeutic targets to alleviate chemoresistance in patients with RCC. 
A previous study demonstrated the inhibitory role of TBOPP against DOCK1, and TBOPP has been reported to display an antitumor role in other types of cancer, such as colon cancer and skin malignant melanoma $(19,20)$. The present study further demonstrated the inhibitory effect of TBOPP on DOCK1, and displayed the role of TBOPP during RCC, suggesting that TBOPP may serve as a chemotherapeutic sensitizer for RCC.

The DOCK family comprises 11 members, which are atypical Rho guanine nucleotide exchange factors (23). Previous studies have revealed the essential roles of DOCK in several cellular processes, including tumorigenesis $(15,24)$. By activating RAC1, DOCK1 displays key functions in cytoskeletal organization, myoblast fusion and phagocytosis (25-27). A number of studies have suggested that DOCK1 is related to tumorigenesis, tumor growth and invasion in several types of cancer, such as glioblastoma and ovarian cancer, implying that DOCK1 plays a vital role in human cancer progression (28-30). Moreover, the biological role of DOCK1 during bladder cancer suggested that downregulation of DOCK1 could sensitize bladder cancer cells to cisplatin by inhibiting the epithelial-mesenchymal transition (12). In addition, DOCK inhibition by enoxaparin could improve the antitumor and antimigration activity of gefitinib during lung cancer (18). However, the role of DOCK1 during RCC has not been previously reported. The results of the present study suggested that DOCK1 expression levels were associated with cisplatin resistance, which was alleviated by DOCK1 inhibition, suggesting that DOCK1 was related to RCC cisplatin resistance.

Although the results indicated that TBOPP sensitized RCC to cisplatin by inhibiting DOCK1, the present study had a number of limitations. Firstly, the present study did investigate the role of DOCK1 and TBOPP in normal kidney cells. Secondly, the effect of TBOPP on the sensitivity of newer, more RCC specific agents, including sorafenib or pazopanib, was not investigated. Thirdly, Tajiri et al (19) reported that DOCK1 expression is driven by oncogenic mutations of RAS; however, the relationship between DOCK1 and RAS was not explored in the present study.

In conclusion, the present study demonstrated that DOCK1 was associated with RCC cisplatin resistance, which was ameliorated by TBOPP. The results suggested that DOCK1 inhibition may serve as a potential therapeutic strategy to alleviate RCC chemoresistance, and TBOPP might serve as a chemotherapeutic sensitizer.

\section{Acknowledgements}

Not applicable.

\section{Funding}

The present study was supported by the National Natural Science Foundation of China (grant no. 81602217).

\section{Availability of data and materials}

The datasets used and/or analyzed during the current study are available from the corresponding author on reasonable request.

\section{Authors' contributions}

LX and WC conceived the study. XZ, SX and SZ performed the experiments. JM, YC and XL analyzed the data. WZ participated in the design of the study and drafted the manuscript. HN conceived the study and revised the manuscript. All authors read and approved the manuscript and agree to be accountable for all aspects of the research in ensuring that the accuracy or integrity of any part of the work are appropriately investigated and resolved.

\section{Ethics approval and consent to participate}

Not applicable.

\section{Patient consent for publication}

Not applicable.

\section{Competing interests}

The authors declare that they have no competing interests.

\section{References}

1. Jonasch E, Gao J and Rathmell WK: Renal cell carcinoma. BMJ 349: g4797, 2014.

2. Rossi SH, Klatte T, Usher-Smith J and Stewart GD: Epidemiology and screening for renal cancer. World J Urol 36: 1341-1353, 2018.

3. Van Poppel H, Becker F, Cadeddu JA, Gill IS, Janetschek G, Jewett MA, Laguna MP, Marberger M, Montorsi F, Polascik TJ, et al: Treatment of localised renal cell carcinoma. Eur Urol 60: 662-672, 2011.

4. Vitale MG, Bracarda S, Cosmai L, Crocetti E, Di Lorenzo G, Lapini A, Mandressi A, Martorana G, Masini C, Montironi R, et al: Management of kidney cancer patients: 2018 guidelines of the Italian Medical Oncology Association (AIOM). Tumori 105 (4_suppl): S3-S12, 2019.

5. Sánchez-Gastaldo A, Kempf E, González Del Alba A and Duran I: Systemic treatment of renal cell cancer: A comprehensive review. Cancer Treat Rev 60: 77-89, 2017.

6. Tanji $\mathrm{N}$ and Yokoyama M: Treatment of metastatic renal cell carcinoma and renal pelvic cancer. Clin Exp Nephrol 15: 331-338, 2011.

7. Yagoda A, Petrylak D and Thompson S: Cytotoxic chemotherapy for advanced renal cell carcinoma. Urol Clin North Am 20: 303-321, 1993.

8. Laurin M and Côté JF: Insights into the biological functions of Dock family guanine nucleotide exchange factors. Genes Dev 28: 533-547, 2014.

9. Morishita K, Anh Suong DN, Yoshida H and Yamaguchi M: The drosophila DOCK family protein Sponge is required for development of the air sac primordium. Exp Cell Res 354: 95-102, 2017.

10. Sanematsu F, Nishikimi A, Watanabe M, Hongu T, Tanaka Y, Kanaho Y, Côté JF and Fukui Y: Phosphatidic acid-dependent recruitment and function of the Rac activator DOCK1 during dorsal ruffle formation. J Biol Chem 288: 8092-8100, 2013.

11. Laurin M, Huber J, Pelletier A, Houalla T, Park M, Fukui Y, Haibe-Kains B, Muller WJ and Côté JF: Rac-specific guanine nucleotide exchange factor DOCK1 is a critical regulator of HER2-mediated breast cancer metastasis. Proc Natl Acad Sci USA 110: 7434-7439, 2013.

12. Chen DJ, Chen W, Jiang H, Yang H, Wang YC and Chen JH: Downregulation of DOCK1 sensitizes bladder cancer cells to cisplatin through preventing epithelial-mesenchymal transition. Drug Des Devel Ther 10: 2845-2853, 2016.

13. Zhang B, Li H, Yin C, Sun X, Zheng S, Zhang C, Shi L, Liu Y and Lu S: Dock1 promotes the mesenchymal transition of glioma and is modulated by MiR-31. Neuropathol Appl Neurobiol 43: 419-432, 2017. 
14. Zhang G, Zhang J, Yang X, Zhang X, Yang S, Wang J, Hu K, Shi J, Ke X and Fu L: High expression of dedicator of cytokinesis 1 adversely influences the prognosis of acute myeloid leukemia patients undergoing allogeneic hematopoietic stem cell transplantation. Cancer Manag Res 11: 3053-3060, 2019.

15. Gadea $\mathrm{G}$ and Blangy A: Dock-family exchange factors in cell migration and disease. Eur J Cell Biol 93: 466-477, 2014.

16. Jarzynka MJ, Hu B, Hui KM, Bar-Joseph I, Gu W, Hirose T, Haney LB, Ravichandran KS, Nishikawa R and Cheng SY: ELMO1 and Dock180, a bipartite Rac1 guanine nucleotide exchange factor, promote human glioma cell invasion. Cancer Res 67: 7203-7211, 2007.

17. Liang Y, Wang S and Zhang Y: Downregulation of Dock1 and Elmo1 suppresses the migration and invasion of triple-negative breast cancer epithelial cells through the RhoA/Racl pathway. Oncol Lett 16: 3481-3488, 2018.

18. Pan Y, Li X, Duan J, Yuan L, Fan S, Fan J, Xiaokaiti Y, Yang H Wang Y and Li X: Enoxaparin sensitizes human non-small-cell lung carcinomas to gefitinib by inhibiting DOCK1 expression, vimentin phosphorylation, and Akt activation. Mol Pharmacol 87: 378-390, 2015

19. Tajiri H, Uruno T, Shirai T, Takaya D, Matsunaga S, Setoyama D, Watanabe M, Kukimoto-Niino M, Oisaki K, Ushijima M, et al: Targeting Ras-driven cancer cell survival and invasion through selective inhibition of DOCK1. Cell Rep 19: 969-980, 2017.

20. Tomino T, Tajiri H, Tatsuguchi T, Shirai T, Oisaki K, Matsunaga S, Sanematsu F, Sakata D, Yoshizumi T, Maehara Y, et al: DOCK1 inhibition suppresses cancer cell invasion and macropinocytosis induced by self-activating Rac1 ${ }^{\mathrm{P} 29 \mathrm{~S}}$ mutation. Biochem Biophys Res Commun 497: 298-304, 2018.

21. Zhang Y, Fang L, Zang Y, Ren J and Xu Z: CIP2A promotes proliferation, invasion and chemoresistance to cisplatin in renal cell carcinoma. J Cancer 9: 4029-4038, 2018.

22. Molina AM, Motzer RJ and Heng DY: Systemic treatment options for untreated patients with metastatic clear cell renal cancer. Semin Oncol 40: 436-443, 2013.
23. Namekata K, Kimura A, Kawamura K, Harada C and Harada T: Dock GEFs and their therapeutic potential: Neuroprotection and axon regeneration. Prog Retin Eye Res 43: 1-16, 2014.

24. Schmidt S and Debant A: Function and regulation of the Rho guanine nucleotide exchange factor Trio. Small GTPases 5: e29769, 2014.

25. Laurin M, Dumouchel A, Fukui Y and Côté JF: The Rac-specific exchange factors Dock1 and Dock5 are dispensable for the establishment of the glomerular filtration barrier in vivo. Small GTPases 4: 221-230, 2013.

26. Laurin M, Fradet N, Blangy A, Hall A, Vuori K and Côté JF: The atypical Rac activator Dock180 (Dock1) regulates myoblast fusion in vivo. Proc Natl Acad Sci USA 105: 15446-15451, 2008.

27. Albert ML, Kim JI and Birge RB: Alphavbeta5 integrin recruits the CrkII-Dock180-rac1 complex for phagocytosis of apoptotic cells. Nat Cell Biol 2: 899-905, 2000.

28. Wang J, Dai JM, Che YL, Gao YM, Peng HJ, Liu B, Wang H and Linghu H: Elmol helps dock180 to regulate Rac1 activity and cell migration of ovarian cancer. Int J Gynecol Cancer 24: 844-850, 2014

29. Li W, Xiong X, Abdalla A, Alejo S, Zhu L, Lu F and Sun H: HGF-induced formation of the MET-AXL-ELMO2-DOCK180 complex promotes RAC1 activation, receptor clustering, and cancer cell migration and invasion. J Biol Chem 293: $15397-15418,2018$

30. Feng H, Hu B, Jarzynka MJ, Li Y, Keezer S, Johns TG, Tang CK, Hamilton RL, Vuori K, Nishikawa R, et al: Phosphorylation of dedicator of cytokinesis 1 (Dock180) at tyrosine residue Y722 by Src family kinases mediates EGFRvIII-driven glioblastoma tumorigenesis. Proc Natl Acad Sci USA 109: 3018-3023, 2012.

This work is licensed under a Creative Commons Attribution-NonCommercial-NoDerivatives 4.0 International (CC BY-NC-ND 4.0) License. 